\title{
System Development for Monitoring Physiological Parameters in Living Environment
}

\author{
Oliver Mladenovski ${ }^{1}$, Jugoslav Achkoski ${ }^{1}$ (D), \\ and Rossitza Goleva ${ }^{2(\triangle)}(\mathbb{D}$ \\ ${ }^{1}$ Military Academy "General Mihailo Apostolski", \\ Skopje, Former Yugoslav Republic of Macedonia \\ oliver.mladenovski@yahoo.com, \\ jugoslav.ackoski@ugd.edu.mk \\ 2 New Bulgarian University, Sofia, Bulgaria \\ rgoleva@gmail.com
}

\begin{abstract}
Nowadays, sensors, algorithms and software applications are embedded in people's everyday lives. To be more precise, living environment is significantly dependent on previously mentioned technology especially in the field of medicine.

The ultimate goal of this research is to collect data from different medical sensors platform, which can be used in initial medical assessment and management in the living environment. Furthermore, collected data from sport's activity were analysed in order to measure the strength of linear association between variables.

First, research on market available equipment is performed which is focused on medical sensors for assisted living in people's everyday environment. Second, research on hardware in terms of tablet, desktop machines, iPad etc. is conducted and scenarios of using the hardware are elaborated. Regarding the scenarios, the concept that demonstrates the advantages and disadvantages of the system for ambient assisted living is shown. The architecture of the system followed by sensors, computer networks, terminals is included and the technology for achieving the system's functionality is presented. Next, the Zephyr Bioharness 3 sensor for measuring physiological data is used for collecting data in the living environment. Finally, integration of the sensor with the most convenient terminal in the living environment is done and collected data are visualized via MATLAB. The parameters monitored are Heart Rate (HR), Respiratory Rate (RR), Peak Acceleration (PkAcc). The proof of the correlation between listed parameters describes the physiological status in the living environment.

The ratio between HR and PkAcc is 0.91. It is a strong positive correlation. It means that changes in heart beats are linearly followed by the peak acceleration. Next, the ratio between HR and RR is 0.88 . It is also a strong positive correlation. It means that changes in heart beats are linearly followed by the respiratory rate. The ratio between RR and PkAcc is 0.84. Again, it is a strong positive correlation. It means that changes in respiratory rate are linearly followed by the peak acceleration.
\end{abstract}


Keywords: Zephyr Bioharness 3 sensor $\cdot$ Smart device

Physiological parameters · Android application development

Data visualization

\section{Introduction}

This chapter contributes in system development for physiological parameters monitoring in living environment. It describes the integration of different technologies in terms of collecting physiological data, algorithms for processing collected data and software application for visualization.

The main problem that this research highlights is the creation of a system that could response on the problems that health caring institutions face nowadays during their primary tasks. The goal is to create a system that could decrease the risk of losing personnel or suffering damage through checking and monitoring of the physiological status. The technological approach for solving a medical problem and assessing a patient's status in ambient living environment is introduced in a more efficient and accurate manner. It means that certain medical algorithms used for initial assessment are researched in the creation of models for assessing (i.e. triage) the medical status. It is based on a Machine Learning (ML) algorithm.

There are many articles that illustrate the use of machine learning algorithms in decision making while assessing the medical status, but the contemporary technological development in terms of hardware, software, operating systems, computer networks, and processing power influence on their extended use in the fields (i.e. initial medical assessment) where they are not sufficiently exploited.

Although ML algorithms are used in numerous medical applications for supporting physicians' decisions, the implementation in medical emergency has not been enough yet. In addition, automated prediction for the patient's status in real-time based on ML algorithm and patient vital signs monitoring supports medical centers preparations for the level of treating patients after pre-hospital triage.

The paper is structured as follows. The second section presents related work in the field. The third section talks about the equipment related to measuring psychophysiological parameters, problems with reports of conducted statistical analysis. The fourth section shows the architecture of the whole system that has being developed. Fifth section presents proof from the field experiment. Sixth section presents the approaches in terms of conducted multiple linear regression analysis and correlation over created dataset. At the end there are future research plan and conclusion.

\section{Related Works}

The paper has been inspired by the needs to have developed system that can collect data from living environment including live streaming monitoring [1]. During the research it has been found that some similar works exists [2]. Hailstone et al. [2] were testing the sensor Zephyr BioHarness ${ }^{\mathrm{TM}}$ and they have done a research with study case and experimental proof. The accuracy of their research motivated the presented implementation in living environment. 
On the other hand, the authors in [3] have compared the accuracy of the BioHarness $^{\mathrm{TM}}$ and Vmax metabolic cart as well as the $\mathrm{K} 4 \mathrm{~b}^{2}$ portable metabolic measurement system in health environment. The present chapter shows a collection of data in different conditions like sport, military exercises, everyday activity. Devices such as the Bioharness $^{\mathrm{TM}}$ are being used in a variety of situations including physical activity, exercise monitoring, and emergency professions. The authors in [4] were testing the dates from the Bioharness in abnormal Heart Rate (HR) conditions which is a one of the expected scenarios in living environment. There are also several authors who are talking about the same topic but they are using some different sensors and methods, like in [5-7]. New generation smart watches have been used to measure the physiological parameters as HR, Galvanic Skin Resistance (GSR), temperature and EQ02 in a multi parameter body-worn system [7]. There are capable to log and transmit the physiological data describing a wearer's cardiorespiratory and thermal status. About the monitoring there are works that describe different techniques actually known to monitor HR. In the $[8,9]$ the authors are focusing in particular on the so-called photo plethysmography (PPG) technique.

An assessment of the reliability of five Bioharness ${ }^{\mathrm{TM}}$ variables using a treadmill based protocol is studied in [10]. The authors also describe the correlation coefficient that are used between the variables.

In [11], an application is developed for improving male/female sports condition dependable on their body profile with the e-health platform. There are some authors that compare different sensors for measuring the physiological parameters on older people. They have found that the creation of an acceptable monitoring device for older persons requires from designers and developers to consider the special demands and abilities of the target group [12-16].

In [17] the authors validate cardiovascular alarms in critically ill patients in an experimental setting by generating a database of physiological data and clinical alarm annotations. They report the current rate of alarms and their clinical validity having collected physiological data at one second intervals while monitoring alarms. It was reported during $982 \mathrm{~h}$ of observation to have 5934 alarms, i.e. six alarms per hour. They find out that about $40 \%$ of all alarms did not correctly describe the patient condition and were classified as technically false; while $68 \%$ of those were caused by manipulation. Only $885(15 \%)$ of all alarms were considered clinically relevant. Most of the generated alarms were threshold alarms $(70 \%)$ and were related to arterial blood pressure $(45 \%)$.

In [18] the authors describe the development of a computer system for long-term large-scale recording and storage of multichannel physiological signals that was built using commercial solutions (software and hardware) and existing hospital IT infrastructure. Both numeric $(1 \mathrm{~Hz})$ and waveform $(62.5-500 \mathrm{~Hz})$ data were captured from 24 Surgical Intensive Care Unit (SICU) bedside monitors simultaneously and stored in a file-based Vital Sign Data Bank (VSDB) during one-year period. The database size is 4.21 TB. Vital signs were recorded from 1175 critically ill patients and up to six Electro Cardiographs (ECG). All type of monitored waveforms and all monitored numeric data were recorded in most of the cases.

Factors and predictors that influence nurses' intention to use the Electronic Intensive Care Unit (eICU) are presented in [19]. The aim is to analyse the applicability of the Technology Acceptance Model in healthcare settings, and to provide psychometric 
evidence of the measurement scales used in the study. The study involved 117 participants from two healthcare systems. The acceptance survey on the use of eICU was conducted as a studying instrument. The results show that perceived usefulness is the most influential factor. The merge between tele-intensive care units, informatics, telecommunication technology, tele nursing, and telemedicine is used in [20] to provide expert, evidence-based, and cutting-edge services to critically ill patients.

The book on enhanced living environments [21] presents the theory and the practice in the field using the up-to-date technological solutions. The main aspects of assistive care systems and applications relevant to the highly positive and dynamic scientific domain have attracted much interest in the world of Internet technology in the last decade.

\section{System Architecture for Collecting Physiological Data}

The purpose of this section is to show the data collection from different multisensory platform, which collect bio signals in terms of ECG, Heart Rate, Respiratory Rate. The architecture is shown in Fig. 1.

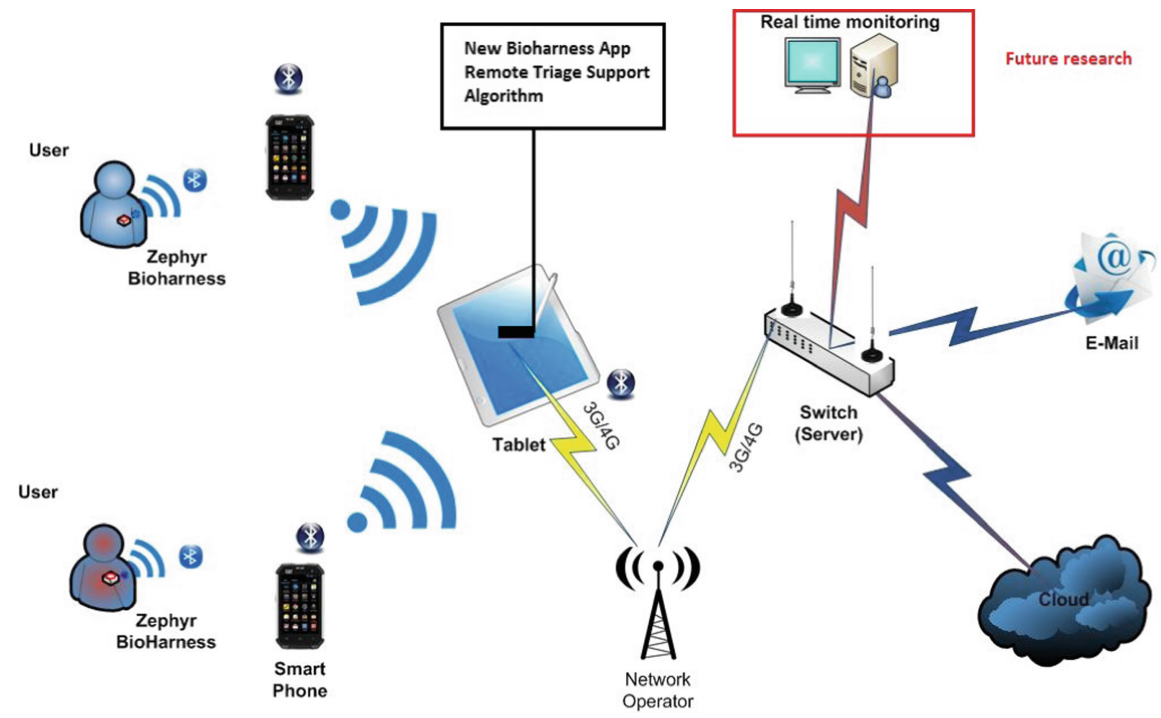

Fig. 1. System structure

The data floating is "wireless" and it is a mobile system with fast data transfer. The use of Bluetooth technology introduced acceptable latency for the measured signals. The data is sent from the user (from the sensor in Fig. 2a) via Bluetooth connection to the smartphone. Also, it can be connected directly to the tablet, where the data is processing by the algorithm that has been used to create the BioHarness App. It could be seen in Fig. $2 b$ and $c$. 


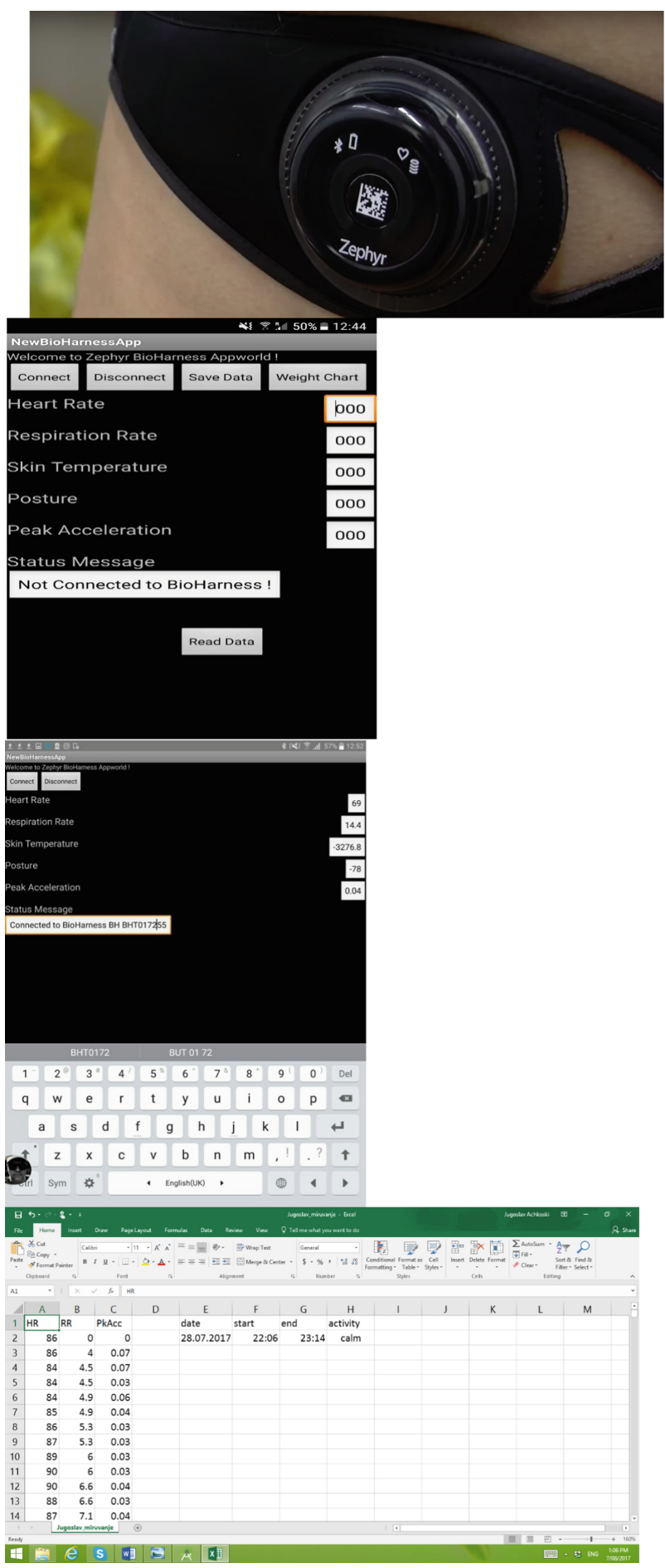

Fig. 2. (a) Zephyr Bioharness 3 sensor; (b) API for Android operating system; (c) Upgraded API; (d) Comma Separated Value (CSV) file 
Processed data via 3G/4G have been transferred to the switch and multicasted to the monitoring center with medical personnel and the cloud.

\section{The Bluetooth Connection}

The Bluetooth technology allows maximum 7 users (devices) to communicate in one piconet (an ad-hoc network) in a master/slave mode. At any time, the data can be transferred between the devices and the master or the "initiator". It decides where the data should be transferred. It is also possible one "slave" to receive data from another "master", i.e. two devices are sending data to one ("slave") at the same time. The technology is being used in industry, Personal Area Networks, smartphones, gaming consoles, Wireless Audio Devices etc. The range and reliability of the systems depend on the firmware, software and hardware of the device but typically it is between $10 \mathrm{~m}$ and $100 \mathrm{~m}$. The last versions support ranges between 40 and $400 \mathrm{~m}$.

Zephyr Bioharness 3BT uses the Bluetooth 1.2+ and the main enhancements on behalf of the other versions are: improved connectivity; better resistance to radio frequency interference; up to $721 \mathrm{kbit} / \mathrm{s}$ transmission speed; improved voice quality of audio links by allowing retransmissions of corrupted packets; better concurrent data transfer; operation with three-wire Universal Asynchronous Receiver-Transmitter (UART); flow control and retransmission modes for logical link control and adaptation protocol (L2CAP).

\section{G/3G Connection}

The information that was gathered from the sensors is transferred to the local switch (server) through the network operator and then to the cloud. It is done after identification of the destination folder. The testing environment is based on the known technologies and use-cases. The review of the six of the most commonly used telemedicine sensors is done, i.e. Isansys, Angel health sensor, Biosignalplux, Opensignal BITalino, Biovotion, e-Health Sensor Platform V2.0 for Arduino and Raspberry Pi. The comparison is based on the type, specifications, certification as a medical device, use of SDK and API, presence of Bluetooth communication or 3G/4G, type of measured vital signs, existence of a cloud and application (Table 1).

Table 1. Review of the market available sensors and their options

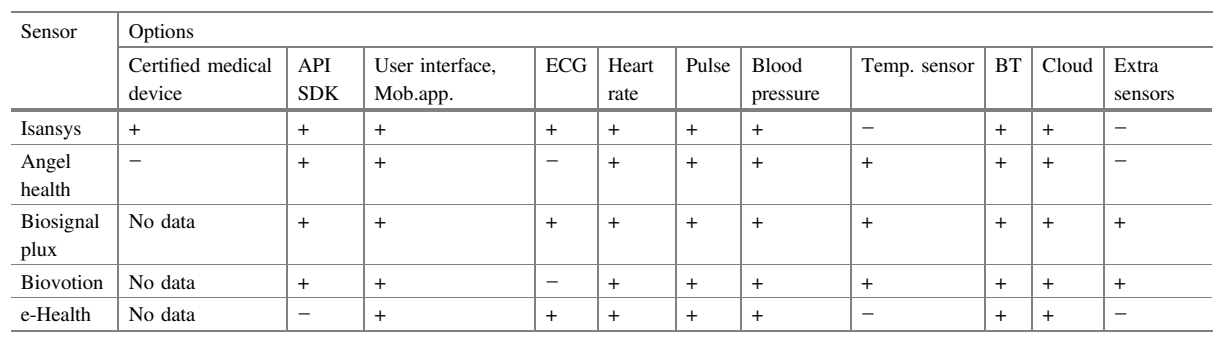

However, abovementioned sensors were not appropriate for ambient living environment. The most convenient biosensor on the market was BioHarness ${ }^{\text {TM }} 3$ (Fig. 2a). It also could be used in different activities (sport, military exercises, everyday activity). 
The BioHarness ${ }^{\mathrm{TM}} 3$ captures and transmits comprehensive physiological data from the wearer to mobile and fixed data networks enabling genuine remote monitoring of human performance and condition in the real world.

Concerning experiment in the lab, the following physiological data can be measured: heart rate, respiratory rate, interbeat (R-R) interval, breathing rate, ECG, posture, activity level, peak acceleration. In terms of communication, the connection between BioHarness ${ }^{\text {TM }} 3$ and DellLatitude 3340 Notebook Laptop Computer is established via Bluetooth communication protocol. Also, the communication between Laptop Computer and Samsung Galaxy S7 is established by Samsung OEM Micro USB 2.0 Charging Cable.

Next, Zephyr API for Android operating system is provided by the manufacturer for collecting physiological data (Fig. 2b). Also, regarding Android based API it does not include support for the ECG in the interface.

Communication protocol for transferring physiological data is Bluetooth 2.0 which supports data transfer of up to $3 \mathrm{Mbit} / \mathrm{sec}$.

The above-mentioned laboratory setup is standardized for Android-based application development.

Because there is no function for storing data from sensor the API is upgraded with additional functionalities such as storing data in CSV file. The physiological data are collected in the following order: heart rate, respiratory rate and peak acceleration. Next, the interface is inflated with three additional buttons: "Save Data", "Weight Chart" and "Read Data" (Fig. 2c).

The "Save Data" button is used to store the data in CSV file (Fig. 2d). It should be stressed that additional methods and variables are added in the class MainActivity.java. Also, AndroidManifest.xml file from API is upgraded with permission for writing data by the following line:

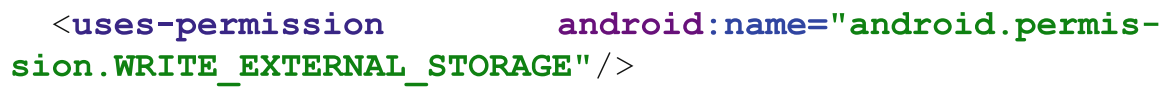

Another two buttons are mock-up buttons and are planned to gain functionalities in further phase of the system development.

\section{Experimental Design}

\subsection{Description}

In addition to laboratory experiment, the field experiment is done. Android J1 PacKage (APK) file is exploited to store physiological data in CSV file. During the experiment, three physiological parameters are collected: heart rate, respiratory rate, and peak acceleration. The case study is created to support field experiment of established communication between a biosensor and a smart device. Also, a new software module is implemented in Zephyr API for storing data into CSV file during testing. 
The case study is done in group of two persons (one male and one female) in different activity. The activities are in the following order: calm, office, and sport's activity. The persons are on age of 36 (female) and 40 (male). Next, the time for conducting measurements is: calm activity - $60 \mathrm{~min}$ (male), office activity - $60 \mathrm{~min}$ (female) and sport's activity $30 \mathrm{~min}$ (male).

The photos from the field experiment are in Fig. 3. The Fig. 3a presents the environment where the female is working on daily basis wearing Zephyr Bioharnes 3 . The Fig. $3 \mathrm{~b}$ presents the male in sport's activity equipped with biosensor which is connected to the smart device.

Every activity is stored by the developed application in separate CSV file. The files, which are generated by application during different activities have the following sizes: calm activity $-83 \mathrm{~KB}$; sport's activity $-27 \mathrm{~KB}$; and office activity $-67 \mathrm{~KB}$.

The sport's activity measured interval is $30 \mathrm{~min}$ (from 08:56 until 09:26 on July 27, 2017). There are 1913 samples recorded in the CSV file.

The obtained records from each activity are plotted in Fig. 4.

\subsection{Methods}

Correlation coefficient technique is used to work up the strength of the relationships and the level of dependence between measured data. The scale applied for the relationship between variable starts with perfect, goes through strong and moderate and ends with weak (Fig. 5).

The $r$ value describes the relation with a number, i.e. it compares things whether they are weak (d), strong (b), moderate (c) or perfect (Fig. 5).

When $\boldsymbol{r}=1$ it is a perfect relationship. When $\mathrm{r}$ is between 0,75 and 0,5 it is a very strong relationship. When there is a moderate relationship the value of $r$ is between 0,5 and 0,75 . When $r$ is between 0,25 and 0,5 it is a weak relationship.

There is a negative correlation when a perfect relationship is present but goes down the hill in the negative direction (Fig. 6).

For the correlations in Fig. 7 the value of $r$ is going to be negative. For example when $\boldsymbol{r}=-1$ it is a perfect relationship.

The difference between positive and negative correlations is that in the positive correlations the values of the independent $(x)$ and the values of the dependent $(y)$ increases, whereas in the negative correlations the values of the independent $(x)$ increases and the value of dependent (y) decreases. Therefore, the value of $r$ is between $\{-1 ; 1\}$.

Additional analysis has been done in order to measure the strength of linear association between two variables. In this test, the Pearson Correlation Coefficient (R) is exploited and the focus is on sport's activity because there have been many variations in variables. 

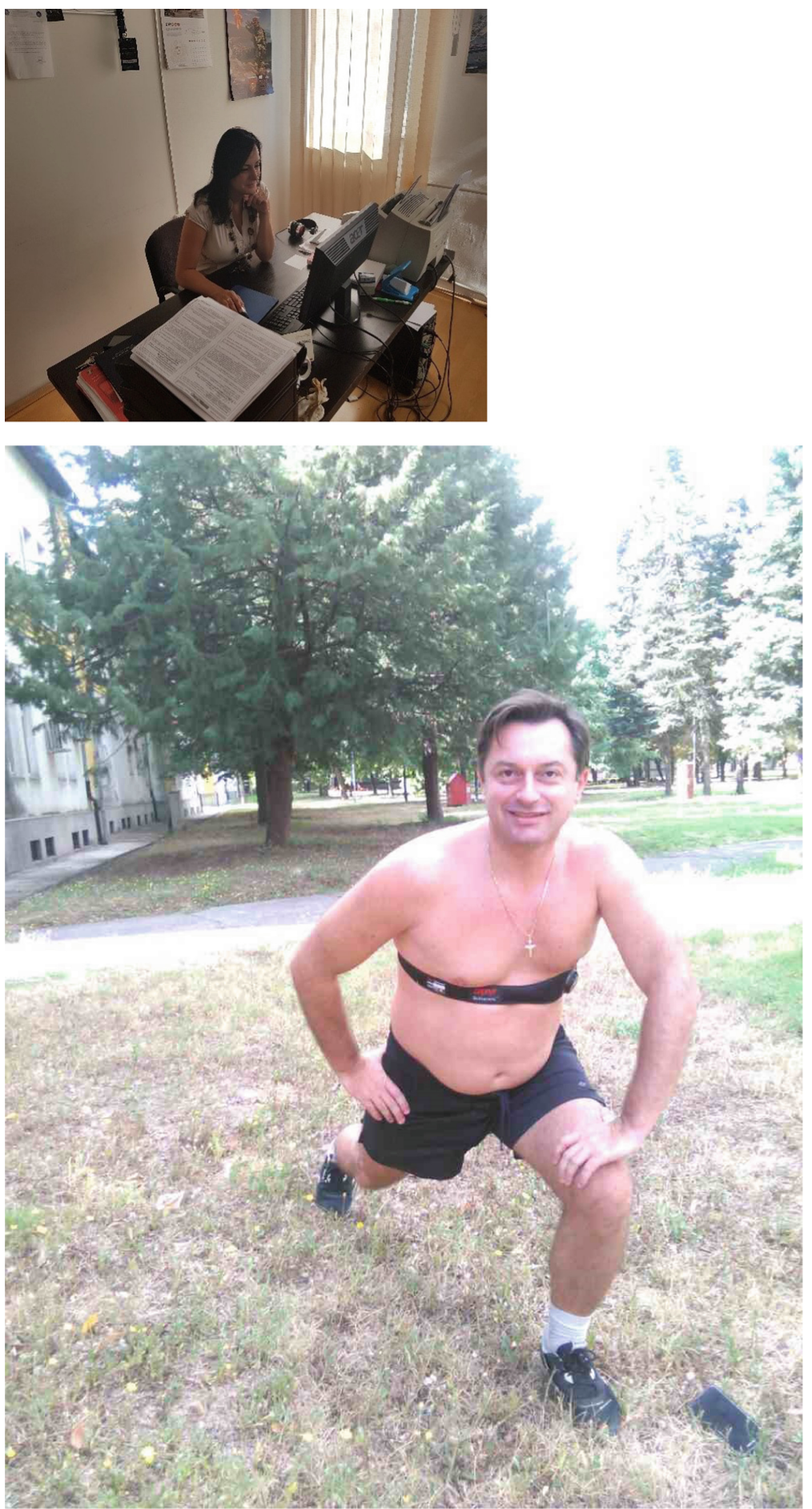

Fig. 3. (a) Activity in office environment; (b) Activity in sport's environment 


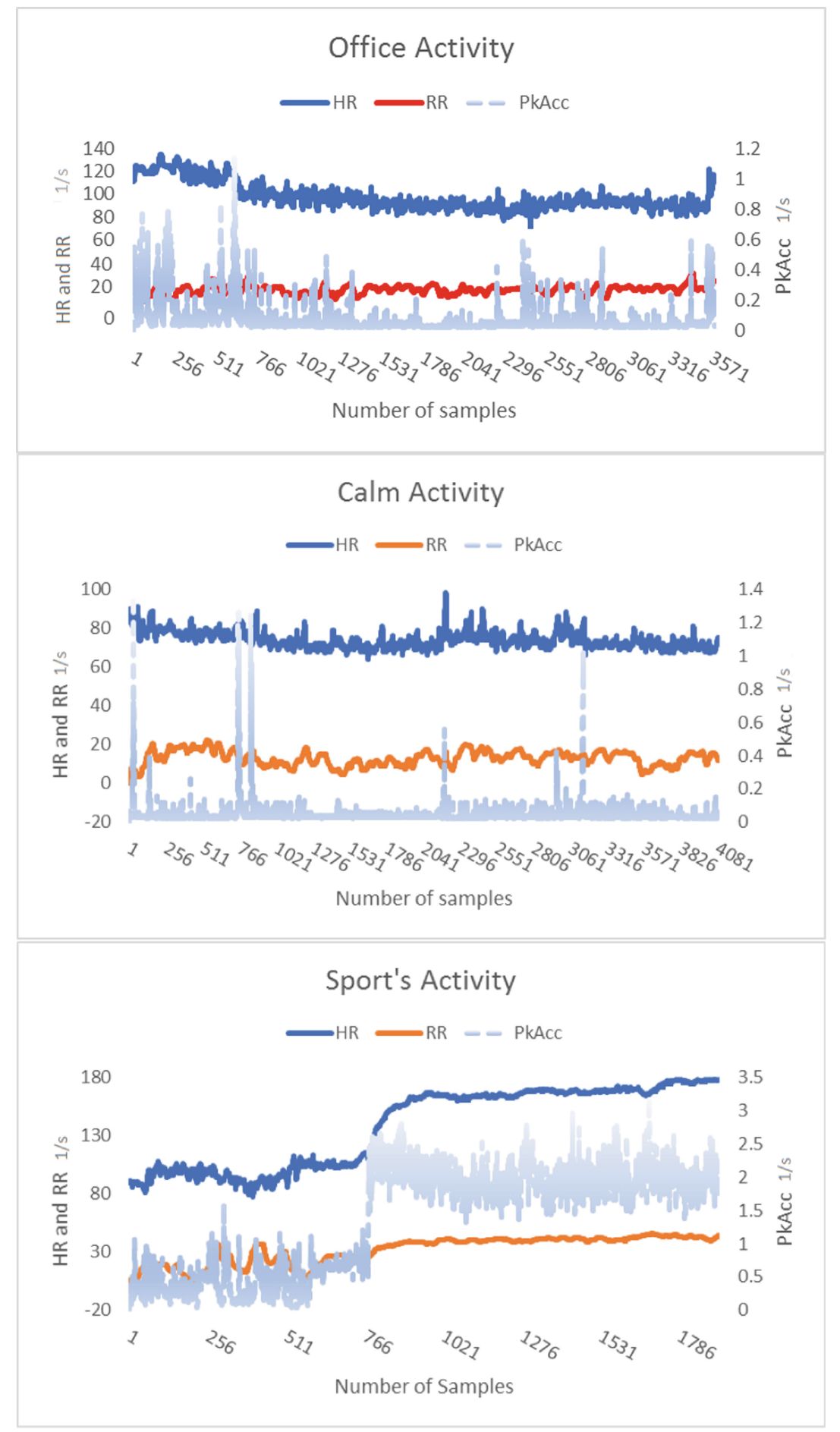

Fig. 4. (a) Office activity; (b) Calm activity; (c) Sport's activity 


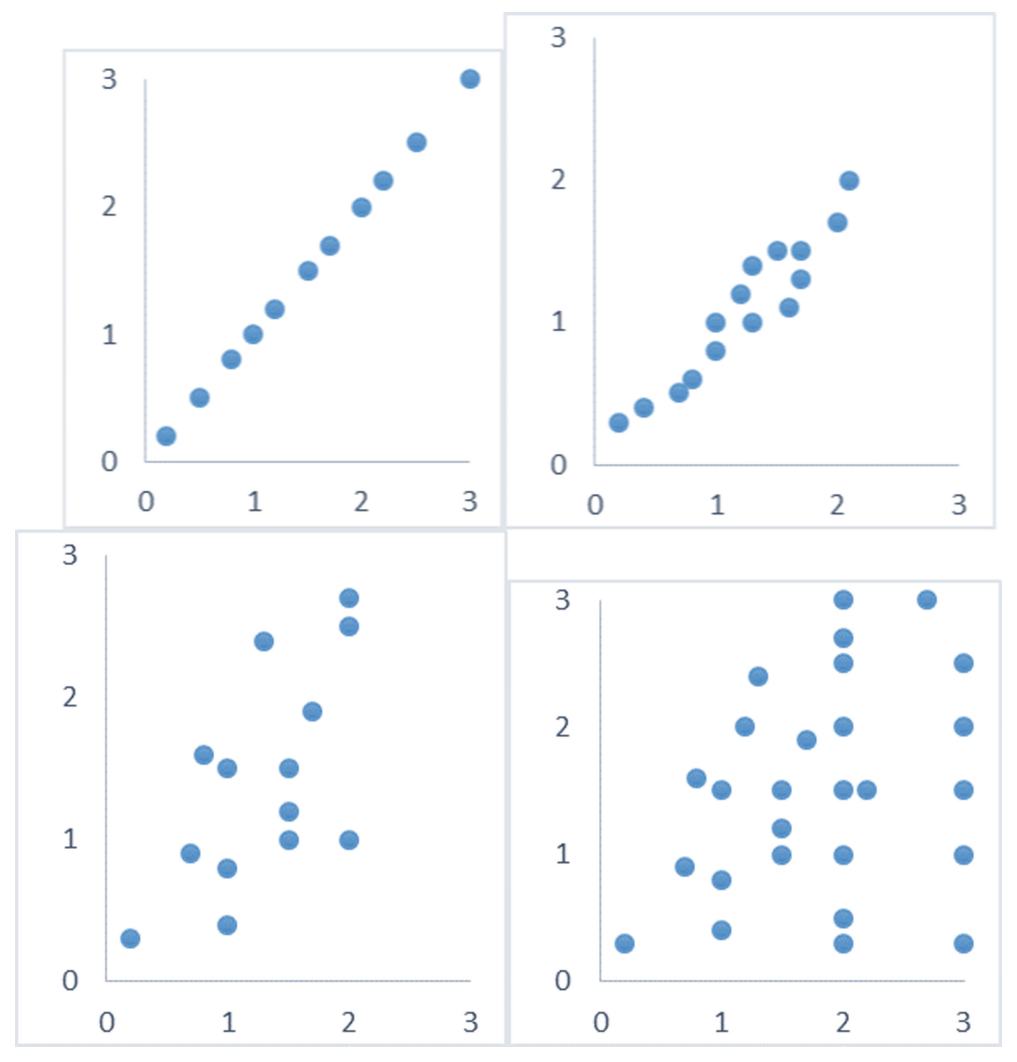

Fig. 5. (a) Perfect positive correlation; (b) Very strong correlation; (c) Moderate correlation; (d) Weak correlation

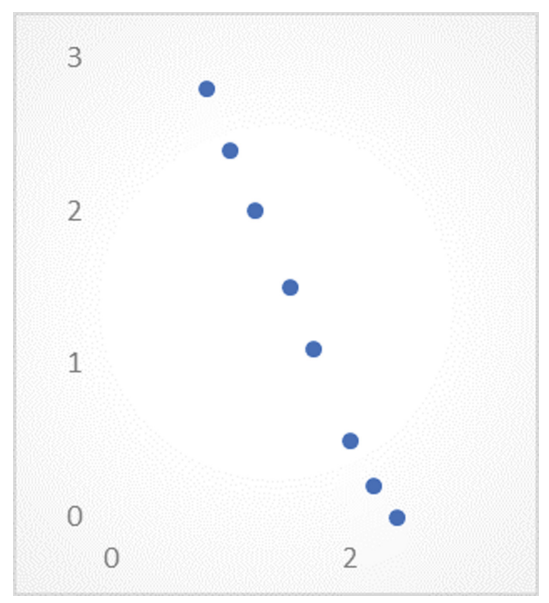

Fig. 6. Perfect negative correlation 


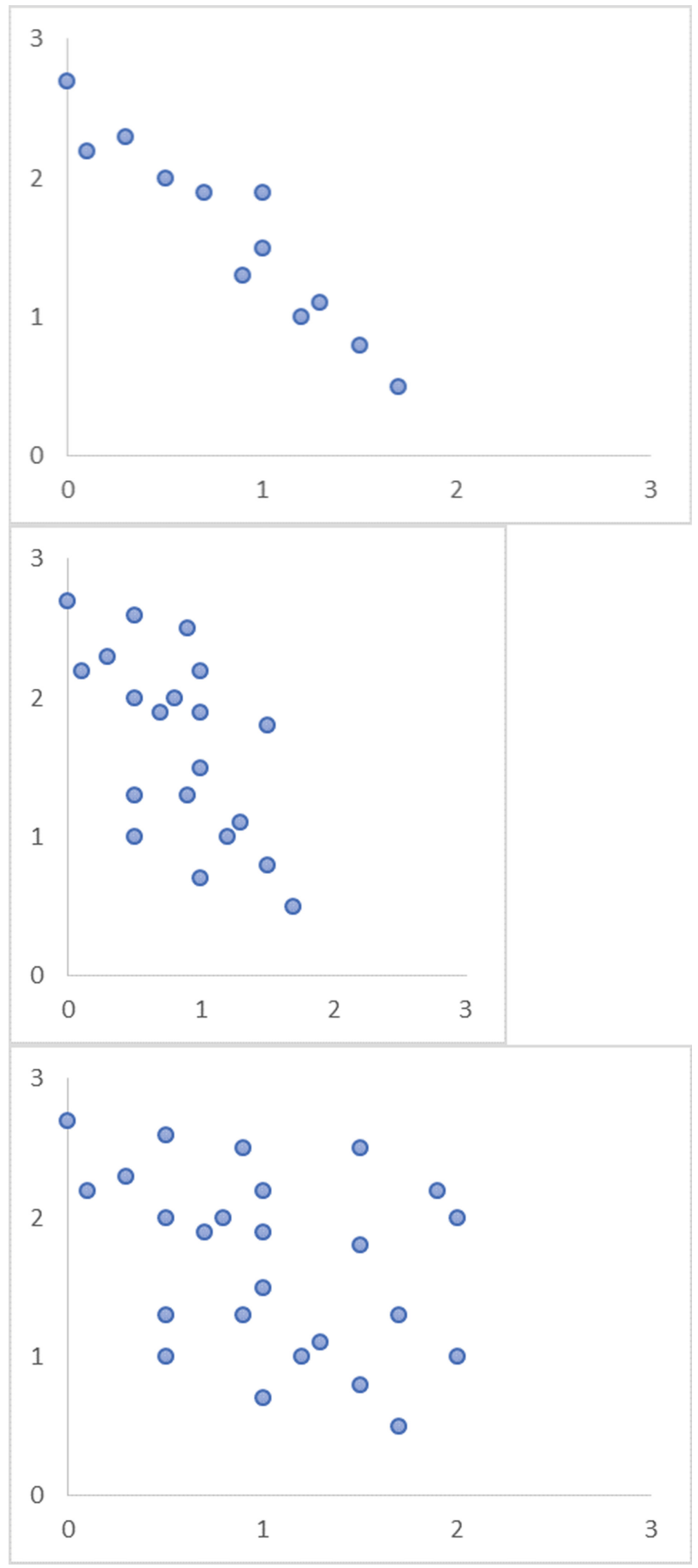

Fig. 7. (a) Very strong correlation; (b) Moderate correlation; (c) Weak correlation 
Firstly, the values from the sensor has been taken for the HR, RR and PkAcc. The correlations between $\mathrm{x}$ and $\mathrm{y}$ is explained by:

$$
r=\frac{n\left(\sum x y\right)-\left(\sum x\right) \times\left(\sum y\right)}{\sqrt{\left[n\left(\sum x^{2}\right)-\left(\sum y\right)^{2}\right]\left[n\left(\sum y^{2}\right)-\left(\sum y\right)^{2}\right]}}
$$

The sum of the values for $(x, y)$ is:

$$
\begin{aligned}
& \sum x=x_{1}+x_{2}+x_{3}+\ldots+x_{n} \\
& \sum y=y_{1}+y_{2}+y_{3}+\ldots+y_{n}
\end{aligned}
$$

Multiplications are:

$$
x y_{1}=x_{1} \times y_{1} ; x y_{2}=x_{2} y_{2} ; x y_{3}=x_{3} \times y_{3} \ldots x y_{n}=x_{n} \times y_{n}
$$

And sum of the values for $x y_{1}, x y_{2} \ldots x y_{n}$ is:

$$
\sum x y_{n}=x y_{1}+x y_{2}+x y_{3}+\ldots+x y_{n}
$$

The following expression has to be found:

$$
x_{1}^{2}+x_{2}^{2}+x_{3}^{2}+\ldots+x_{n}^{2}
$$

And

$$
\begin{gathered}
y_{1}^{2}+y_{2}^{2}+y_{3}^{2}+\ldots+y_{n}^{2} \\
\sum x^{2}=x_{1}^{2}+x_{2}^{2}+x_{3}^{2}+\ldots+x_{n}^{2} \\
\sum y^{2}=y_{1}^{2}+y_{2}^{2}+y_{3}^{2}+\ldots+y_{n}^{2}
\end{gathered}
$$

\subsection{Results}

In Fig. 8 the scatter plots are created so as the correlation between measured physiological parameters to be visualized.

In Fig. 8a the correlation between the heart rate and peak acceleration is shown where the $\mathrm{HR}$ is in the $\mathrm{X}$ axis and the peak acceleration is on the $\mathrm{Y}$ axes. The ratio (value of R) between HR and PkAcc is 0.91 . It is a strong positive correlation. It means that changes in heart beats are linearly followed by peak acceleration.

In Fig. 8b the correlation between heart rate and respiratory rate is presented with RR in $\mathrm{X}$ axis and HR in $\mathrm{Y}$ axes. The value of $\mathrm{R}$ between HR and RR is 0.88 and demonstrates a strong positive correlation. It means that changes in heart beats are linearly followed by respiratory rate. 


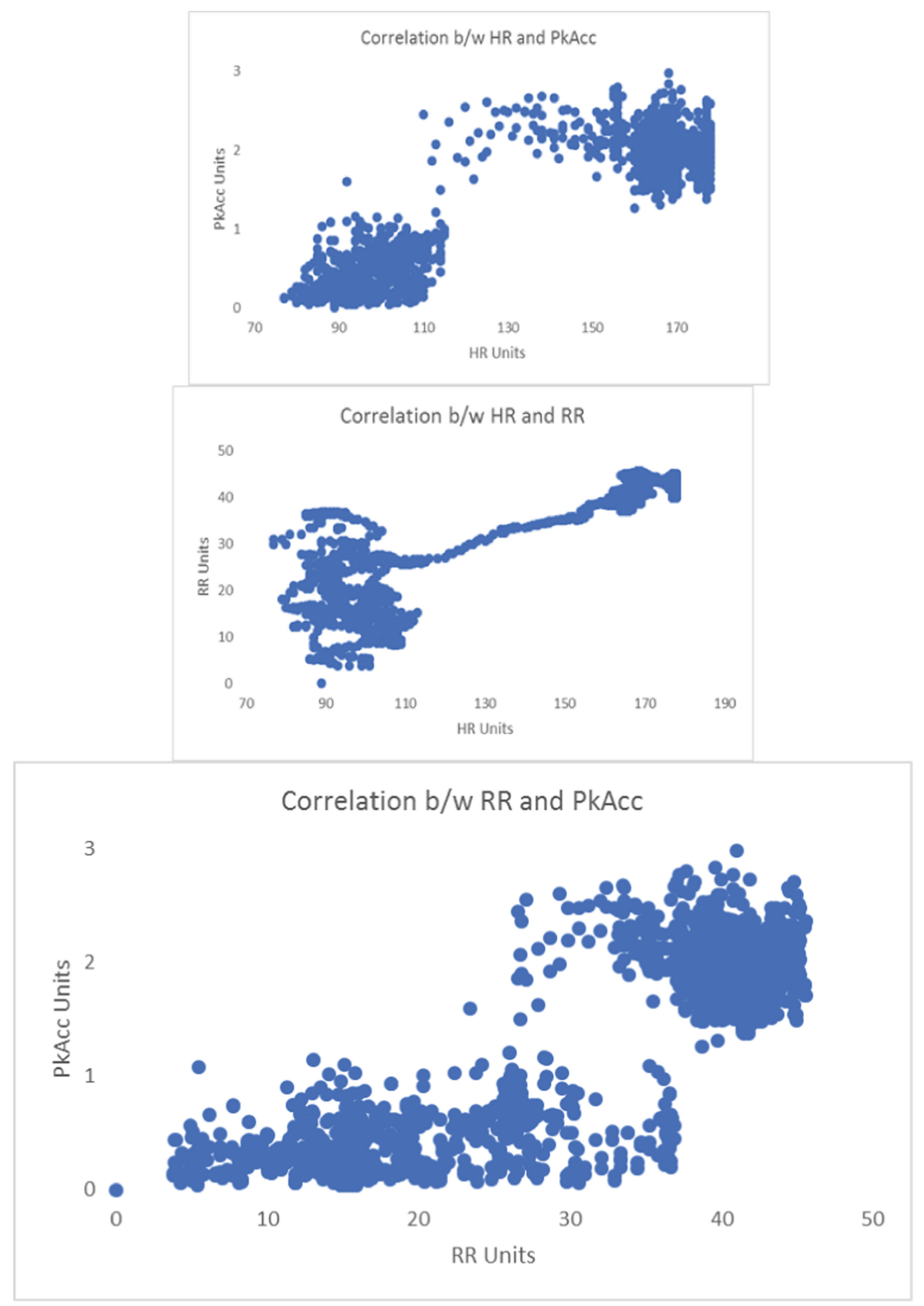

Fig. 8. (a) Correlation between HR and PkAcc; (b) Correlation between HR and RR; (c) Correlation between RR and PkAcc 
In Fig. 8c there is a correlation between RR and Pkacc where RR is in $\mathrm{X}$ axis and PKAcc in $\mathrm{Y}$ axes. The value of $\mathrm{R}$ between $\mathrm{RR}$ and PkAcc is 0.84 showing a strong positive correlation. It means that changes in respiratory rate are linearly followed by peak acceleration.

\section{Conclusion}

The obtained results about correlation of psychophysiological parameters are very strong. It means that changes in heart beats are linearly followed by peak acceleration and respiratory rate. The tests need to be performed also by different groups of older adults looking for the changes in correlation results related to the age and disease.

The system will be extended with two new modules in the developed Android application adding a fuzzy logic algorithm as a decision instrument to raise an alert. In addition, the collected data is going to be visualized using the CSV file, monitoring center, live streaming module and cloud services.

Acknowledgement. Our thanks to COST Action IC1303: Algorithms, Architectures and Platforms for Enhanced Living Environments (AAPELE).

\section{References}

1. Johnstone, J.A., et al.: BioHarness ${ }^{\mathrm{TM}}$ multivariable monitoring device: part. I: validity. J. Sport. Sci. Med. 11(3), 400 (2012)

2. Hailstone, J., Kilding, A.E.: Reliability and validity of the Zephyr ${ }^{\mathrm{TM}}$ BioHarness $^{\mathrm{TM}}$ to measure respiratory responses to exercise. Meas. Phys. Educ. Exerc. Sci. 15(4), 293-300 (2011)

3. Kim, J.-H., et al.: Measurement accuracy of heart rate and respiratory rate during graded exercise and sustained exercise in the heat using the Zephyr BioHarness ${ }^{\mathrm{TM}}$. Int. J. Sports Med. 34(06), 497-501 (2013)

4. Boudet, G., Chamoux, A.: Heart rate monitors and abnormal heart rhythm detection. Arch. Physiol. Biochem. 108(4), 371-379 (2000)

5. Jovanov, E.: Preliminary analysis of the use of smartwatches for longitudinal health monitoring. In: 2015 37th Annual International Conference of the IEEE Engineering in Medicine and Biology Society (EMBC). IEEE (2015)

6. Markova, V., Ganchev, T.: Technological support to stress-level monitoring. In: Enhanced Living Environments: From Models to Technologies (Healthcare Technologies 2017), Chap. 6, pp. 133-160. IET Digital Library (2017). https://doi.org/10.1049/pbhe010e_ch6

7. Ruskova, I.N., Gieva, E.E.: Sensors for wireless body area networks. In: Enhanced Living Environments: From Models to Technologies (Healthcare Technologies 2017), Chap. 8, pp. 183-205. IET Digital Library (2017). https://doi.org/10.1049/pbhe010e_ch8

8. Lemay, M., et al.: Application of optical heart rate monitoring. In: Wearable Sensors: Fundamentals, Implementation and Applications, pp. 105-129. Elsevier (2014)

9. Liu, Y., et al.: Validity and reliability of multiparameter physiological measurements recorded by the Equivital LifeMonitor during activities of various intensities. J. Occup. Environ. Hyg. 10(2), 78-85 (2013) 
10. Johnstone, J.A., et al.: Bioharness ${ }^{\mathrm{TM}}$ multivariable monitoring device: part. II: reliability. J. Sport. Sci. Med. 11(3), 409 (2012)

11. Castillejo, P., et al.: Integration of wearable devices in a wireless sensor network for an Ehealth application. IEEE Wirel. Commun. 20(4), 38-49 (2013)

12. Ehmen, H., et al.: Comparison of four different mobile devices for measuring heart rate and ECG with respect to aspects of usability and acceptance by older people. Appl. Ergon. 43(3), 582-587 (2012)

13. Redondi, A., et al.: An integrated system based on wireless sensor networks for patient monitoring, localization and tracking. Ad Hoc Netw. 11(1), 39-53 (2013)

14. Pires, I.M., Garcia, N.M., Pombo, N., Flórez-Revuelta, F., Spinsante, S., Teixeira, M.C.: Identification of activities of daily living through data fusion on motion and magnetic sensors embedded on mobile devices. Pervasive Mob. Comput. 47, 78-93 (2018). https://doi.org/10. 1016/j.pmcj.2018.05.005. ISSN 1574-1192

15. Jovanov, E., Milosevic, M., Milenković, A.: A mobile system for assessment of physiological response to posture transitions. In: 2013 35th Annual International Conference of the IEEE Engineering in Medicine and Biology Society (EMBC). IEEE (2013)

16. Borodin, A., et al.: Architectural approach to the multisource health monitoring application design. In: 2015 17th Conference of Open Innovations Association (FRUCT). IEEE (2015)

17. Siebig, S., Kuhls, S., Imhoff, M., Gather, U., Schölmerich, J., Wrede, C.E.: Intensive care unit alarms - how many do we need? Crit. Care Med. 38(2), 451-456 (2010)

18. Burykin, A., Peck, T., Buchman, T.G.: Using "off-the-shelf" tools for terabyte-scale waveform recording in intensive care: computer system design, database description and lessons learned. Comput. Methods Programs Biomed. 103(3), 151-160 (2011)

19. Kowitlawakul, Y.: The technology acceptance model: predicting nurses' intention to use telemedicine technology (eICU). CIN: Comput. Inform. Nurs. 29(7), 411-418 (2011)

20. Williams, L.M., Hubbard, K.E., Daye, O., Barden, C.: Telenursing in the intensive care unit: transforming nursing practice. Crit. Care Med. 32(6), 62-69 (2012)

21. Dobre, C., Garcia, N., Goleva, R.I., Mastorakis, G.: Ambient Assisted Living and Enhanced Living Environments: Principles, Technologies and Control. Butterworth-Heinemann, Amsterdam (2016)

Open Access This chapter is licensed under the terms of the Creative Commons Attribution 4.0 International License (http://creativecommons.org/licenses/by/4.0/), which permits use, sharing, adaptation, distribution and reproduction in any medium or format, as long as you give appropriate credit to the original author(s) and the source, provide a link to the Creative Commons licence and indicate if changes were made.

The images or other third party material in this chapter are included in the chapter's Creative Commons licence, unless indicated otherwise in a credit line to the material. If material is not included in the chapter's Creative Commons licence and your intended use is not permitted by statutory regulation or exceeds the permitted use, you will need to obtain permission directly from the copyright holder.

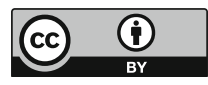

